\title{
Carnot cycle in practice: compensating inefficiencies of ORC expanders through thermal regeneration
}

\author{
Lucie Lefebvre ${ }^{1 *}$, Ward De Paepe ${ }^{1}$, Mario L. Ferrari ${ }^{2}$, Alberto Traverso ${ }^{2}$ \\ ${ }^{1}$ Thermal Engineering and Combustion Unit, University of Mons (UMONS), Belgium \\ ${ }^{2}$ TPG-DIME, University of Genoa, Italy
}

\begin{abstract}
The Organic Rankine Cycle (ORC) is a thermodynamic cycle that can operate with a hot source over a wide range of temperatures, especially with low-grade heat (below $200^{\circ} \mathrm{C}$ ). One of the main limitations for the success of small-scale ORC cycles (few to $100 \mathrm{~kW}_{\mathrm{e}}$ ) is the relatively low isentropic efficiency of the typically used turbomachinery. Low turbine efficiency leads to low ORC cycle performance. To increase the performance of the cycle, the turbine efficiency must be increase, however, this significantly increases the cost of the machinery, making the cycle less profitable. In this work, the performance evaluation of low-temperature ORC cycles $\left(100-150^{\circ} \mathrm{C}\right)$ with heat extraction along the expansion process is investigated, in an attempt to overcome this limitation. The studied cycle works in the same way as a conventional ORC, except that during the expansion process, heat is extracted. This heat is re-used later in the cycle, just before the hot source, allowing to reduce its load. The different cycles presented in this paper, using pentane as working fluid, are compared based on their exergetic and energetic efficiencies. The influence of three parameters on the cycle performance is studied: the regeneration ratio, the maximum temperature of the cycle and the turbine isentropic efficiency. In the case of a cycle using pentane with a maximum temperature of $150{ }^{\circ} \mathrm{C}$ and an turbine isentropic efficiency of $65 \%$, the energy efficiency increases from $6.2 \%$ to $16.3 \%$ when going from no regeneration to full regeneration, and the exergy efficiency increases from 21.1 to $45.8 \%$.. Secondly, the influence of the maximum temperature of the cycle is studied. Using pentane as the working fluid, the higher the maximum temperature is, the larger the benefits of heat extraction. However, this temperature cannot exceed the critical temperature of the organic fluid to stay in the case of a subcritical cycle. Finally, considering the turbine isentropic efficiency, it is possible to demonstrate that using a less efficient turbine, for example in small ORC systems, the performance of a cycle with an ideal turbine isentropic efficiency (100\%) can be achieved compensating at cycle level the turbine losses with the heat extraction along the expansion process.
\end{abstract}

\section{Introduction}

Nowadays, energy consumption is growing as well as the environmental concerns. In addition to this, fossil fuel, the most widespread source of energy, is a finite source. More and more people are becoming aware of these problems and wish for a greener future. In addition, the European Union fixed to reduce the greenhouses gases before 2050 [1]. Hence it is important to increase the share of renewable energy and decrease the fuel consumption by raising the efficiency of energy production processes.

One way to increase efficiency is by recovering the waste heat of industrial processes. M. Papapetrou et al. [2] estimate the total waste heat in Europe around $300 \mathrm{TWh} /$ year, with a third being low-grade heat, i.e. less than $200^{\circ} \mathrm{C}$. A common way to recover waste heat is by using thermodynamic cycles. This work presents a study of the performance of one of them: The Organic Rankine Cycle, noted ORC, and in particular, an ORC using a turbine allowing for heat extraction.

The interest in ORCs has increased significantly in the last two decades [3] and nowadays, the worldwide cumulated installed capacity of this technology is estimated around 2.7 GW [4]. In comparison, the cumulative installed capacity for wind energy is equal to $650.8 \mathrm{GW}$ [5], highlighting that there is much potential for future installations, especially for small-scale applications. Several examples of these kind of applications are available in literature and are summarized in the review presented by Rahbar et al. [7],describing the different cycles, including biomass fired combined heat and power, solar power cycles, geothermal binary power cycles, internal combustion engines waste heat recovery, gas and steam power cycle

\footnotetext{
* Corresponding author: Lucie.Lefebvre@student.umons.ac.be
} 


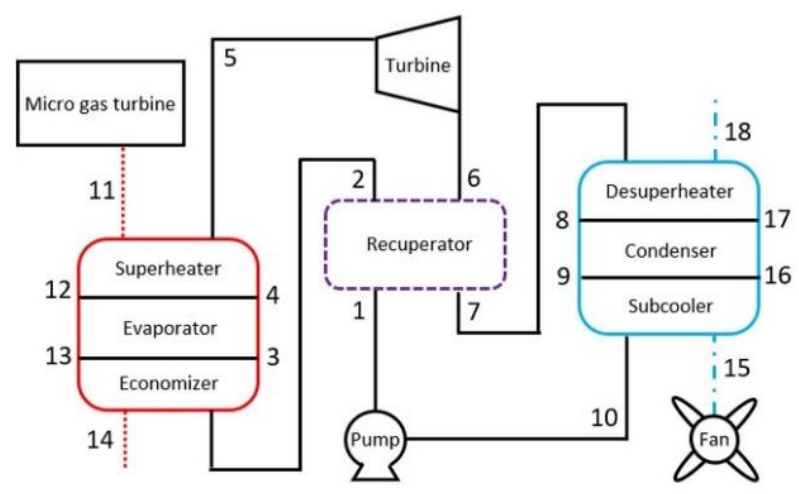

(a)

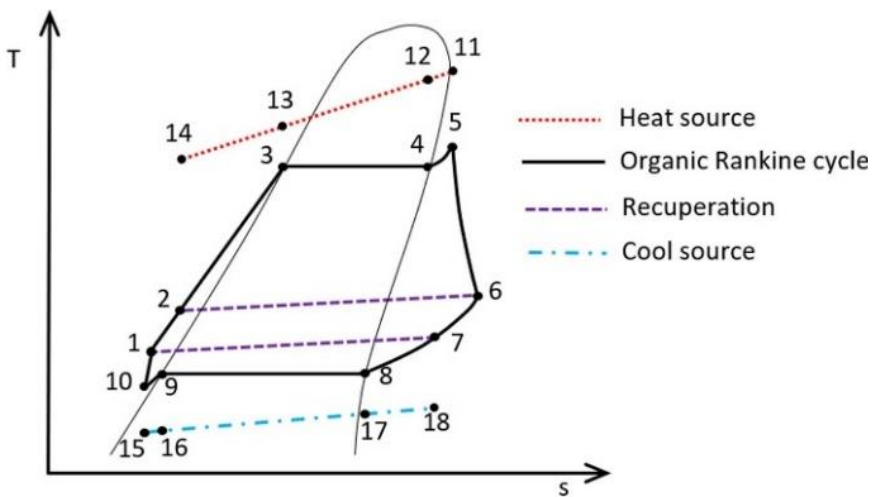

(b)

Fig. 1: Schematic representation of an ORC with a recuperator and the associated $T$-s diagram with Pentane as the working fluid [7].

exhaust heat recovery and heat thermal energy conversion with ORC.

However, the performance of the ORC is currently still too limited, and it is mainly the isentropic efficiency of the expander that has a significant impact on the performance of the cycle [6].

The objective of this work is to assess the performance of a specific Organic Rankine Cycle. Its specificity is the extraction of heat during the expansion process. This extracted heat is re-

used in the cycle to preheat the working fluid being heated further by the hot source. With this heat extraction during the expansion phase, we aim to increase the global cycle performance, allowing to compensate the losses due to the rather low turbine efficiency.

\section{Working principle}

The Organic Rankine Cycle is a thermodynamic cycle using an organic fluid as the working fluid composed of four main steps: (1) exchange with the hot source (preheating, evaporation, and superheating), (2) expansion, (3) exchange with the cold source (desuperheating, condensation and subcooling), and finally, (4) the pressurization in the pump. The Temperature vs entropy diagram and the schematic representation of the cycle are shown in Fig. 1.

In the specific ORC studied in this work, heat is extracted during the expansion process. This heat is reinjected later in the cycle just before the heat source. Therefore, a new point is added to the cycle, point $2^{\prime}$. The extracted heat is reinjected between points 2 and $2^{\prime}$ and point 2 ' corresponds to the economizer input.

\section{Model Description}

To carry out the performance analysis presented in this paper, a previously developed model from de Campos et al. was used and modified [8]. The main change to this code is the extraction of heat during the expansion and the use of this heat later in the same cycle. The amount of heat extracted is quantified by a parameter called the regeneration ratio, noted $\mathrm{R}$, which is equal to the heat recovered during the expansion process divided by the maximum heat load of the economizer. Another difference with the previous model is that in the new model, the code works no matter the state of the working fluid at the end of the expansion whereas before, it only worked for dry expansion.

To simulate heat extraction in the expander, it is necessary to discretize the expansion. Each step of the discretization is divided into two phases: an adiabatic expansion followed by an isobaric heat extraction. Fig. 2 shows the succession of the two phases of the expansion. By increasing the number of steps, it is possible to tend toward a continuous process. Finally, all the cycles studied are scaled to a net output power of $10 \mathrm{~kW}$ to enable an objective comparison.

The performances indicators used in this work are the energy and exergy efficiencies of the cycle. The first is defined as the net power output divided by the power available as energy (Eq.1), and the second is the net power output divided by the power available as exergy (Eq.2).

$\eta_{\text {energy }}=\frac{P_{\text {net }}}{\dot{m}_{O R C}\left(h_{5}-h_{2}\right)}$
$\eta_{\text {exergy }}=\frac{P_{\text {net }}}{\dot{m}_{O R C}\left(e_{5}-e_{2}\right)}$

\section{Results}

In this result section, the impact of three main parameters, being the regeneration ratio, the maximum temperature of the cycle, and the isentropic efficiency of

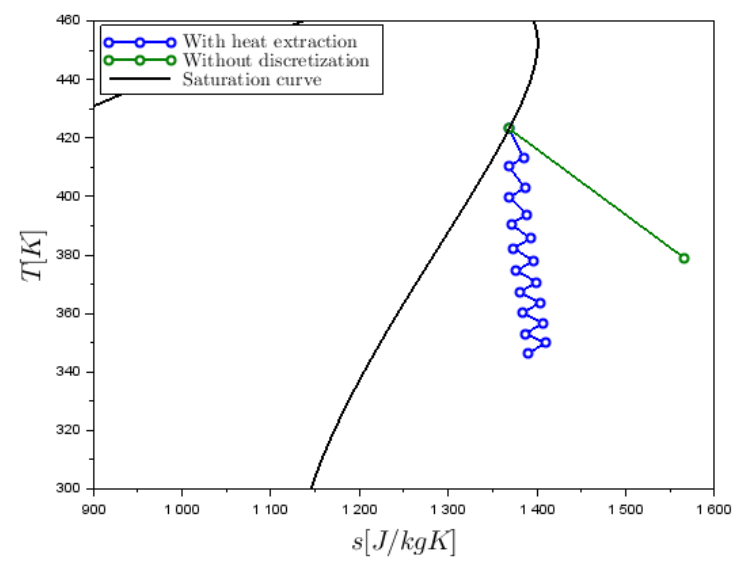

Fig. 2: To simulate the heat extraction expansion process, this process was discretization in an expansion and heat extraction step. 


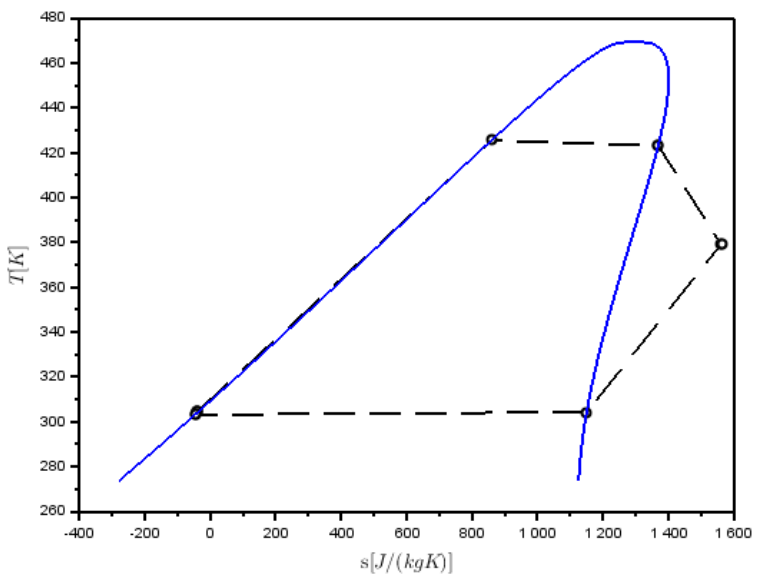

Fig. 3: $T$-s diagram for $R=0$

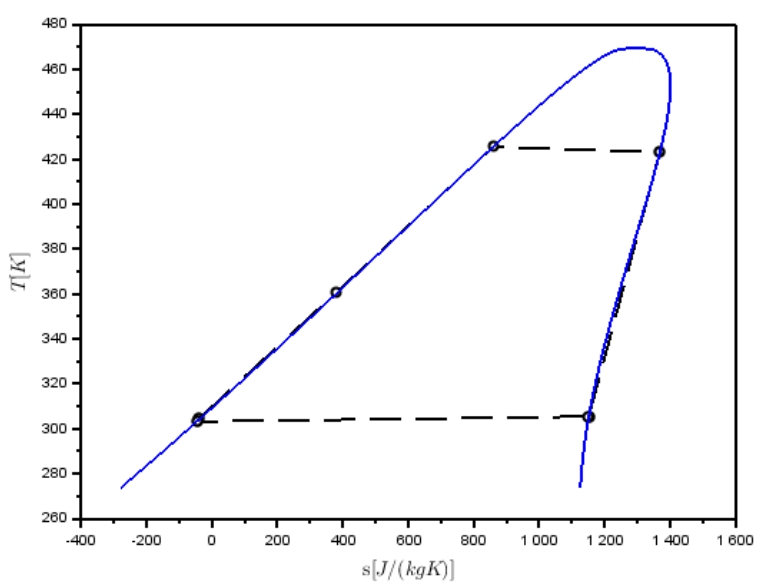

Fig. 4: $T$-s diagram for $R=0.425$

the turbine is assessed and discussed. For all the following cycles, the used working fluid was pentane.

\subsection{Regeneration ratio}

The first studied parameter is the regeneration ratio. When its value increases, the amount of heat extracted during the expansion is more important. Fig. 3, 4 and 5 show the T-s diagrams for increasing values of the regeneration ratio. The first corresponds to a $\mathrm{R}$ equals to 0 , i.e. no heat extraction. Then, when the regeneration ratio increases, point 2', corresponding to the inlet of the economizer, is moving up. When $\mathrm{R}$ is equal to 1 , point 2 ' and 3 coincide, meaning that the economizer is no more required in the cycle. Another observation is that, when $\mathrm{R}$ is equal to 0.425 (Fig. 4), the expansion line is along the saturation curve. It means that when $\mathrm{R}$ is equal or higher to this value, the recuperator is no more needed in the cycle. Additionally, when $\mathrm{R}$ is higher than 0.425 , the expansion ends inside the saturation curve, i.e. in a biphasic state.

By increasing the regeneration ratio, it is possible to increase the energy and exergy efficiency of the cycle (Fig. 6). The values added to the points in Fig. 6 correspond to the vapor quality at the end of the expansion. These results allow to conclude that both exergy and energy efficiencies increase sharply between $\mathrm{R}=0$ and $\mathrm{R}=1$. Indeed, the first one increases from $21.09 \%$ to $45.84 \%$, while the latter one increases from

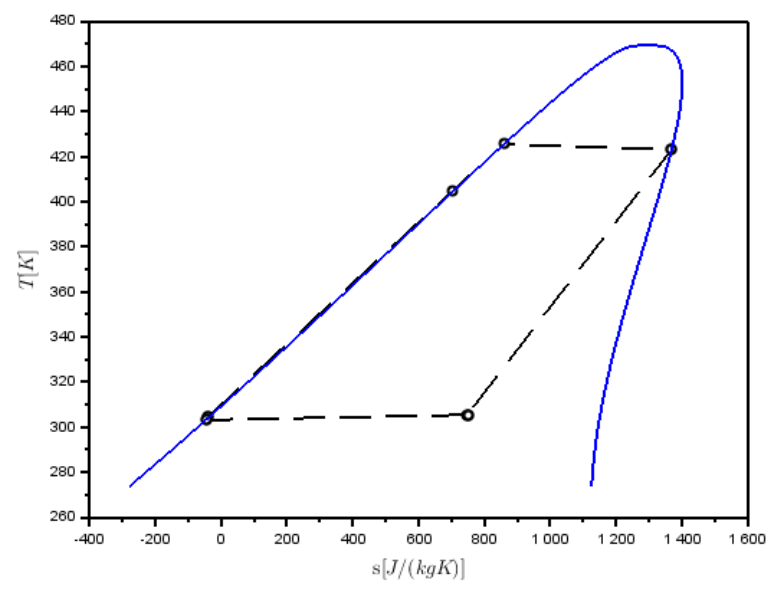

Fig. 5: $T$-s diagram for $R=1$

$6.16 \%$ to $16.25 \%$. The exergy efficiency is doubled, and the energy efficiency is almost tripled, which shows indeed the potential of the heat extracted ORC cycle.

\subsection{Maximum temperature}

The second studied parameter is the maximum temperature of the cycle. Three maximum temperature are considered: $100^{\circ} \mathrm{C}, 125^{\circ} \mathrm{C}$, and $150^{\circ} \mathrm{C}$. Fig. 7 and 8 show, respectively, the evolution of exergy efficiency and energy efficiency for these maximum temperatures. Looking at the energy efficiency evolution, when the regeneration ratio increases, a maximal efficiency is reached for higher maximum temperatures. However, the exergy efficiency has different behaviour. It shows that for $\mathrm{R}=0$, a lower temperature is more convenient in terms of exergy. However, when $\mathrm{R}$ increases, this trend is reversed.

\subsection{Turbine Isentropic Efficiency}

The last studied parameter is the isentropic efficiency of the expander. The interest of this part is to understand, as mentioned in the introduction, if it is possible to achieve the same cycle performance with a low-efficiency turbine by compensating with heat

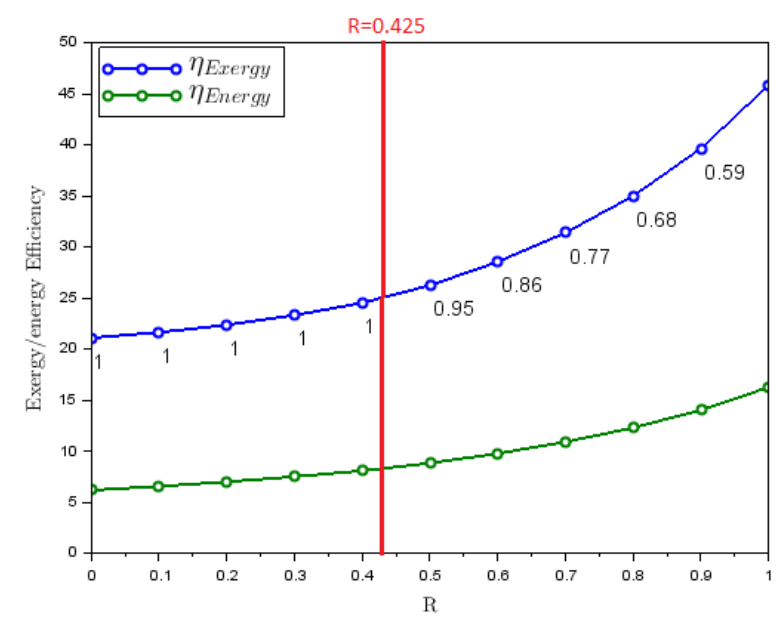

Fig. 6: Evolution of exergy efficiency and energy efficiency with $R$ 


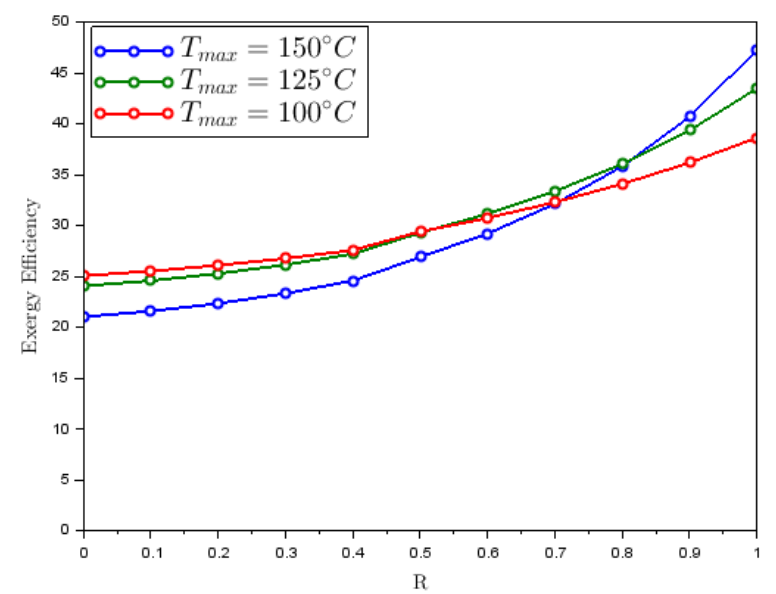

Fig. 7: Exergy efficiency for different maximum temperature

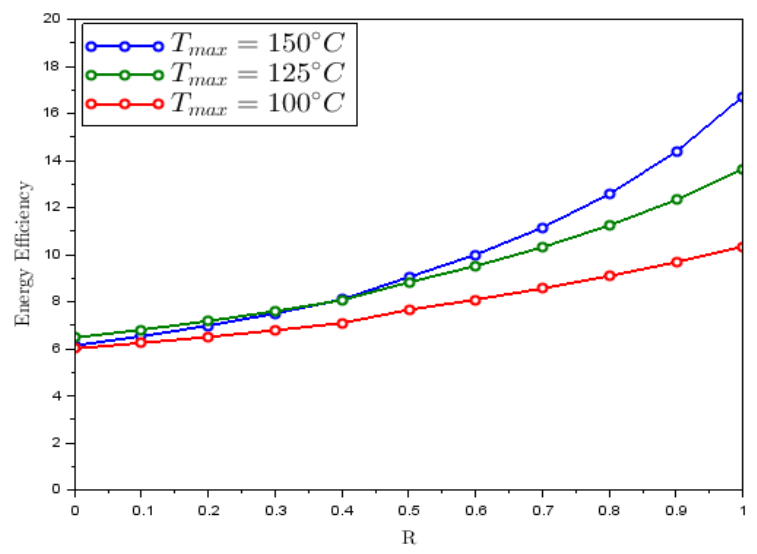

Fig. 8: Energy efficiency for different maximum temperature

extraction. Fig. 9 and 10 show the exergy and energy efficiency variation for different isentropic efficiencies.

First, looking at the exergy evolution (Fig. 9), for an isentropic efficiency of $100 \%$, the exergy efficiency achieved is not equal to $100 \%$. This means that there are also exergy losses in the other components, apart from the turbine, and most probably in the condenser. These losses decrease considerably as the regeneration ratio increases due to the reduced load on the condenser. Simulation results also shows that with a turbine with an isentropic efficiency of, for example, $60 \%$, it is possible to achieve the same cycle performance, i.e. an energy efficiency equal to 10.9 , as with a turbine with $100 \%$ efficiency by increasing the regeneration ratio from 0 to 0.79 . This observation is interesting since it means that by using a turbine with mediocre efficiency, but which allows heat extraction, it is possible to achieve good cycle performance as well.

Then, looking at the energy evolution (Fig. 10), the same conclusion can be draw. The difference with the previous graph is a higher increase between the minimum and maximum value of $\mathrm{R}$ for energy efficiency than for exergy efficiency.

Using Fig. 10, showing the energy efficiency evolution for different isentropic efficiency, three cases are highlighted. These cases represent cycles providing the same energy efficiency with different isentropic efficiency of the turbine. Table 1 lists the main output parameters of the different cases. To obtain the same energy efficiency of $10.9 \%$, the decrease in isentropic efficiency must be balanced by an increase in the

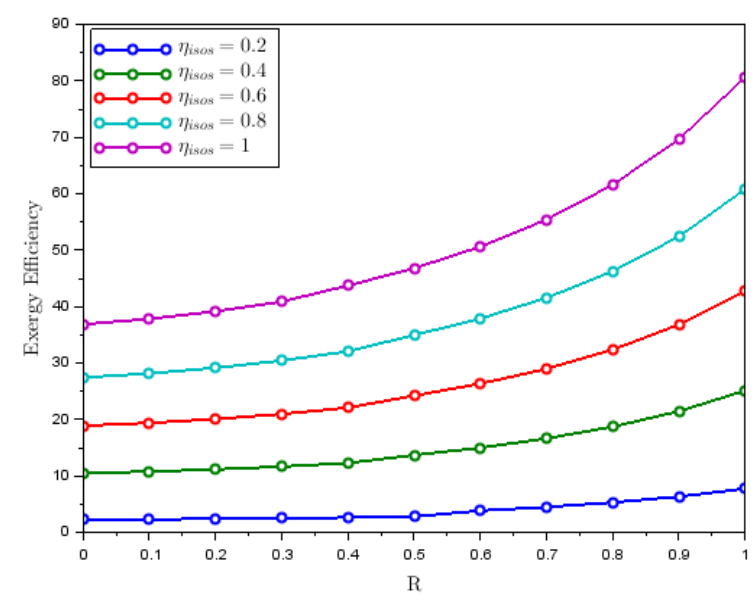

Fig. 9: Exergy efficiency for different isentropic efficiencies.

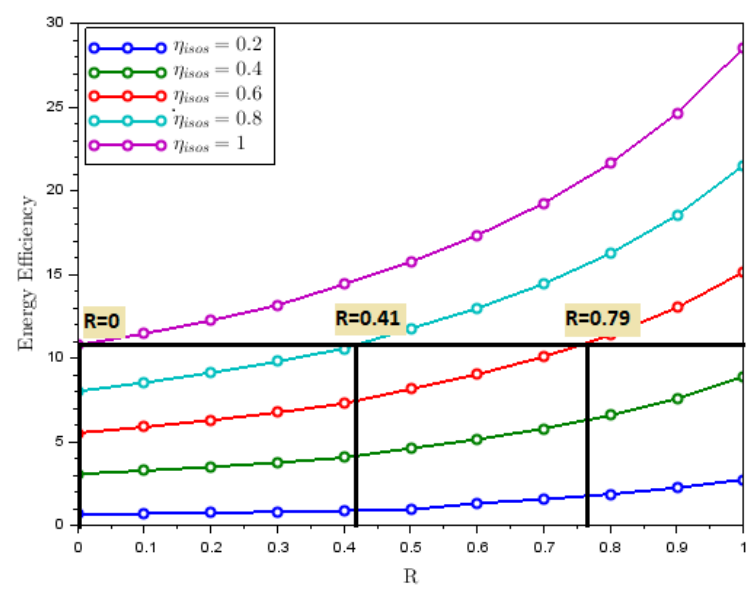

Fig. 10: Energy efficiency for different isentropic efficiencies.

regeneration ratio. In other words, the amount of heat extracted during expansion must increase to keep the same performance. The table also confirms the steeper increase in the energy efficiency compared to the exergy efficiency. The net power is the same in all the cases due to the scaling of the cycles explained before.

In conclusion, if heat extraction during expansion leads to a decrease in expander efficiency, it is still possible to achieve better performance than without heat extraction. In addition, when the regeneration ratio increases, the load on the condenser and evaporator decreases. Finally, when $\mathrm{R}$ is greater than 0.425 , the recuperator is no longer necessary, thus avoiding one component.

Table 1. Main output parameters for the three cases

\begin{tabular}{|c|c|c|c|}
\hline & $\mathbf{1}^{\text {st }}$ case & $\mathbf{2}^{\text {nd }}$ case & $3^{\text {rd }}$ case \\
\hline$\eta_{\text {isos }}[\%]$ & 100 & 80 & 60 \\
\hline$\eta_{\text {energy }}[\%]$ & 10.9 & 10.9 & 10.9 \\
\hline$\eta_{\text {exergy }}[\%]$ & 37.2 & 33.2 & 31.2 \\
\hline $\mathrm{R}$ & 0 & 0.41 & 0.79 \\
\hline$P_{\text {Net }}[k W]$ & 10 & 10 & 10 \\
\hline$P_{\text {gross }}[k W]$ & 11.7 & 12.2 & 12.6 \\
\hline
\end{tabular}




\section{Conclusion}

The objective of this work was to simulate an Organic Rankine Cycle with heat extraction in the expander and recovery of this heat, showing its potential to increase cycle performance. To assess this performance, an existing numerical model was updated, enabling the simulation of the simultaneous heat extraction expansion process. The results show that this approach allows to improve the performances of the ORC. Indeed, the energy efficiency increases from $6.2 \%$ to $16.3 \%$ from no regeneration to full regeneration, and the exergy efficiency increases from 21.1 to $45.8 \%$. Moreover, it has been proven that is possible to achieve the performances of classic ORC with an ORC using a less efficient turbine if heat is recovered from the expansion.

Future work involves the actual development of the heat extraction and regeneration in the cycle components, which is rather challenging, given the small-scale, limited number of stages and hence small lengths and surfaces. 3D printing technology can offer here a possible solution.

\section{Acknowledgement}

This project has received funding from the European Union's Horizon 2020 research and innovation programme under grant agreement

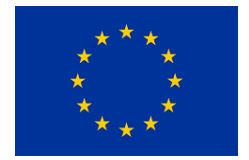
No 764706, PUMP-HEAT.

\section{References}

1. M. Rivarolo, A. Cuneo, A. Traverso, A.F. Massardo. Design optimisation of smart polygeneration energy districts through a model based approach. Applied Thermal Engineering, 99 (2016) 291-301.

2. M. Papapetrou, G. Kosmadakis, A. Cipollina, Umberto, and G. Micale. Industrial waste heat: Estimation of the technically available resource in the eu per industrial sector, temperature level and country. Applied Thermal Engineering, 138:207 $216(2018)$

3. Y-P. Park, M. Usman, M. Imran, and A. Pesyridis. Review of organic rankine cycle experimental data trends. Energy Conversion and Management, 173:679-691 (2018)

4. T. Tartière, M. Astolfi. A world overview of the organic rankine cycle market.Energy Procedia, 129:2 - 9 (2017)

5. Jean-Daniel Pitteloud. Global wind installations. https://public.tableau.com (2019)

6. M. Ibarra, A. Rovira, D-C. Alarcón-Padilla, J. Blanco. Performance of a $5 \mathrm{kWe}$ Organic Rankine Cycle at part-load operation. Applied Energy, 120:147-158 (2014)

7. K. Rahbar, S. Mahmoud, R. K. Al-Dadah, N. Moazami, and S.A.Mirhadizadeh. Review of organic rankine cycle for small-scale applications.
Energy Conversion and Management, 134:135 155 (2017)

8. G. B. de Campos, C. Bringhenti, A. Traverso, and J-s. Takachi Tomita. Thermoeconomic optimization of organic rankine bottoming cycles for micro gas turbines. Applied Thermal Engineering, 164:114477 (2020)

9. S. Lecompte, H. Huisseune, M. van den Broek, B. Vanslambrouck, and M. De Paepe. Review of organic rankine cycle (orc) architectures for wasteheat recovery. Renewable and Sustainable Energy Reviews, 47:448 - 461, 2015. 